\title{
ORIGINAL
}

\section{EVOLUCIÓN DE LA MORTALIDAD INFANTIL, NEONATAL y POSTNEONATAL EN ANDALUCÍA, 1975-1998}

\author{
Miguel Ruiz Ramos (1) y María Adoración Nieto García (2)
}

(1) Consejería de Salud, Junta de Andalucía, Sevilla

(2) Departamento de Ciencias Sociosanitarias. Facultad de Medicina. Sevilla.

\section{RESUMEN}

Fundamento: La mortalidad infantil, y sus componentes neonatal y postneonatal, son importantes indicadores de salud, lo cual justifica su análisis periódico incluso en países desarrollados donde sus tasas han descendido considerablemente. El objetivo de este estudio es describir las modificaciones registradas en dichas tasas en Andalucía en el último cuarto de siglo.

Métodos: Se han calculado las tasas anuales de mortalidad infantil, neonatal precoz y tardía, así como post-neonatal en 1975 1998. Mediante regresión de Poisson se han estimado los porcentajes anuales de cambio de las tasas en los dos intervalos de 1975-1986 y 1987-1998 así como en el periodo completo 1975-1998. Se ha analizado también la mortalidad proporcional por causas infecciosas, respiratorias, congénitas, afecciones originadas en el periodo perinatal y el resto de causas, así como la razón de tasas de mortalidad por afecciones originadas en el periodo perinatal y para el total de causas, en el quinquenio 1994-98 respecto al quinquenio 1975-79, en los periodos infantil, neonatal (precoz y tardío) y postneonatal.

Resultados: Los mayores descensos porcentuales se han producido en la mortalidad neonatal precoz $(6,38 \%)$ y tardía $(4,6 \%)$. La razón de tasas de mortalidad por afecciones originadas en el periodo perinatal entre 1994-98 y 1975-79 es 10 para la mortalidad postneonatal mientras que es inferior a 1 para la mortalidad neonatal tardía $(0,63)$, precoz $(0,33)$ e infantil $(0,30)$.

Conclusiones: La mortalidad en los periodos infantil, neonatal precoz, tardío y post-neonatal ha descendido acusadamente entre 1975 y 1998. El riesgo de fallecer por afecciones originadas en el periodo perinatal de los niños con más de 4 semanas y menos de 1 año de vida (periodo postneonatal) se ha multiplicado por diez entre $1975-79$ y $1994-98$.

Palabras clave: Mortalidad infantil. Mortalidad neonatal. Mortalidad postneonatal. Tendencias temporales de mortalidad.

Correspondencia:

Miguel Ruiz Ramos

Instituto de Estadística de Andalucía

Pabellón de Nueva Zelanda, Leonardo Da Vinci s/n

Isla de la Cartuja, 41071 Sevilla

Correo electrónico: miguel.ruiz.ext@juntadeandalucia.es

\section{ABSTRACT}

\section{Infant, neonatal and postneonatal mortality trends in Andalucía, Spain, 1975-1989}

Background: Infant mortality and its neonatal and postneonatal aspects are important health indicators and thus warrant regular analysis even in developed countries where the rates thereof have dropped considerably. This study is aimed at describing the changes recorded in these rates in Andalusia over the past twenty-five years.

Methods: The annual infant, early and late neonatal and postneonatal mortality rates have been calculated for the 1975-1998 period. Poisson regression was used to estimate the annual percentages of change in the rates for the 1975-1986 and 1987-1998 periods, as well as for the entire 1975-1998 period. An analysis was also made of the proportional mortality rate due to infectious, respiratory, congenital causes, disorders having arisen during the perinatal period and all other causes, as well as the ratio for mortality rates due to disorders having arisen in the perinatal period and for all causes as a whole for the 1994-1998 four-year period as compared to the 19751979 period, in infant, neonatal (early and late) and postnatal periods.

Results: The greatest percentage drops were in early (6.38\%) and late $(4.6 \%)$ neonatal mortality. The ratio for mortality rates due to disorders having arisen in the perinatal period for the 1994-1998 and $1975-1975$ periods is 10 for the postneonatal mortality rate, whilst it is under 1 for the late $(0.63)$ and early $(0.33)$ neonatal and infant $(0.30)$ mortality.

Conclusions: Mortality during the infant, early and late neonatal and postneonatal periods dropped sharply during the 1975-1998 period. The risk of death due to disorders arising during the perinatal period among children ranging from four weeks to one year of age (postneonatal period) rose tenfold during the 1975-1979 and 19941998 periods.

Key words: Infant mortality. Neonatal mortality. Time-related trends in mortality. 


\section{INTRODUCCIÓN}

La mortalidad infantil se ha considerado uno de los indicadores más útiles para valorar el estado de salud de una comunidad ${ }^{1}$. De hecho se estima que las mejoras en la alimentación, en la vivienda, en las condiciones de trabajo y de vida de la población en general, así como en el nivel educativo de las madres en particular, han sido los principales factores implicados en el descenso de las tasas de mortalidad infantil registrado en los países desarrollados, de forma paulatina y continuada, a lo largo del siglo $\mathrm{XX}^{2,3}$.

Esta reducción de las defunciones en el primer año de vida respecto al número de nacidos vivos ha sido más marcada en aquellos países de Europa que, como España y Portugal, partían de tasas de mortalidad infantil más elevadas. El último informe Eurostat $^{4}$ presentaba una tasa de mortalidad infantil en España en 1998 de 5,7 por 1000, cercana a la tasa promedio europea $(5,2)$, eliminando las importantes diferencias que separaban las tasas de España y Europa en los años 60 y 70.

Sin embargo, esta reducción no ha sido de igual intensidad a lo largo del tiempo ni en cada intervalo de los primeros 12 meses de vida del recién nacido. En los últimos años, a medida que las cifras de mortalidad infantil alcanzaban valores más bajos, se enlentecía el proceso de reducción de la mortalidad infantil en general, especialmente a partir de las primeras cuatro semanas de vida del recién nacido. Este hecho se ha puesto de manifiesto en trabajos de ámbito regional ${ }^{5}$, nacional $^{6}$ e internacional ${ }^{7,8}$.

Estos cambios han coincidido con un descenso muy importante de la tasa de fecundidad, que en el ámbito nacional se sitúa actualmente por debajo de la tasa de recambio poblacional ${ }^{9}$, y que presenta, como la mortalidad infantil, una distribución geográ- fica desigual. De hecho, las Comunidades Autónomas menos desarrolladas presentan descensos algo menos marcados en la fecundidad y mantienen también tasas más elevadas de mortalidad infantil ${ }^{10}$.

El objetivo de este trabajo es describir la evolución que la mortalidad infantil, neonatal precoz, neonatal tardía y postneonatal han experimentado entre 1975 y 1998 en la Comunidad Autónoma de Andalucía de Andalucía, con una población total actual de 7.300.000 de habitantes.

\section{MATERIAL Y MÉTODOS}

Las defunciones anuales de los menores de un año y los nacidos vivos se han obtenido de las publicaciones del Instituto Nacional de Estadística (INE) ${ }^{11}$ y del Instituto de Estadística de Andalucía (IEA) ${ }^{12}$, de 1975 a 1979 las causas de mortalidad fueron clasificadas según la $8^{\mathrm{a}}$ revisión de la Clasificación Internacional de Enfermedades (CIE) y el resto de los años por la $9^{\text {a }}$ revisión. Se han calculado las tasas anuales de mortalidad infantil, neonatal precoz, tardía y postneonatal de 1975 a 1998. El periodo neonatal, como recoge la CIE $10^{a}$ revisión ${ }^{13}$, comienza en el nacimiento y termina 28 días completos después del nacimiento; se han subdividido en muertes neonatales precoces, que ocurren durante los siete primeros días de vida, y las muertes neonatales tardías, que ocurren después del séptimo día pero antes de los 28 días completos de vida; y el periodo postneonatal se ha considerado desde los 28 días hasta el año de vida.

Mediante modelos de regresión de Poisson ${ }^{14}$ se estimaron los porcentajes de cambio anuales (PCA), de las tasas en los periodos 1975-1986, 1987-1998 y 1975-1998, el software utilizado ha sido EGRET, los PCA se han calculado mediante la diferencia entre uno y los riesgos relativos (RR) estimados por los modelos de regresión, es decir un RR de 1,40 se interpreta como un PCA de $+40 \%$ 
y un RR de 0,60 como un PCA de $-40 \%$. Se ha utilizado el periodo de 1975 a 1998 por ser el primer y último año disponible de mortalidad por causas clasificadas con dos revisiones de la CIE relativamente comparables $^{15}$ y se han dividido en dos periodos iguales de 12 años. La variable independiente fue el año de defunción y la dependiente la tasa de mortalidad. Se han construido modelos para la mortalidad infantil y cada uno de sus componentes, así como para la mortalidad por causas perinatales, en este caso se agregaron los años de defunción en periodos de cinco años excepto para 1990-1993 que fueron cuatro.

Para describir el cambio producido en las causas de mortalidad a estas edades se han utilizado el primer y último quinquenio disponible, por un lado, por ser los periodos más alejados y por tanto dan una información más clara respecto al objetivo del trabajo, y por otro, se han agregado en quinquenios para disminuir la varianza del indicador. El criterio que se ha utilizado para la lista de causas ha sido que la agrupación de causas que incluyera todas las defunciones de los menores de un año en el primer periodo. En los quinquenios de 1975 -79 y 1994-98 se han calculado la mortalidad proporcional por causa de defunción agrupando las siguientes categorías de la revisión de la Clasificación Internacional de Enfermedades (CIE): infecciosas (CIE-8: 000-136,CIE-9 000-139), respiratorias (CIE-8 y CIE-9: 460-519), congénitas (CIE-8 y CI9-9: 740-759), afecciones originadas en el periodo perinatal (CIE-8 y CIE9: 760-779) y otras causas (rúbricas CIE-8 y CIE-9) no incluidas en las agrupaciones anteriores).

Por último, se han calculado las razones de las tasas de mortalidad por afecciones originadas en el periodo perinatal y por todas las causas entre los quinquenios inicial y final (1975-1979 y 1994-1998) en el periodo infantil, neonatal precoz y tardío y postneonatal.

\section{RESULTADOS}

La tabla 1 muestra el número de nacidos vivos, las defunciones y las tasas de mortalidad infantil y sus componentes en Andalucía para cada uno de los años del periodo estudiado, así como el porcentaje de cambio anual estimado para dicho periodo y la primera y segunda mitad del mismo. El número de nacidos vivos se ha reducido en más de un $38 \%$ en el conjunto del periodo. El descenso producido en la mortalidad infantil desde 1975 a 1998 ha sido de 21,66 defunciones por 1000 nacidos vivos en 1975 a 5,76 en 1998, lo que supuso un PAC de -5,62\%. Esta tendencia se produjo de forma desigual entre los distintos componentes de la mortalidad infantil, la neonatal precoz fue la que más descendió con un PAC de -6,38, seguida de la neonatal tardía y la postneonatal con un descenso del 4,6\% y 4,35\% respectivamente (ver figura 1). La magnitud del descenso durante la primera y segunda mitad del periodo fue diferente según la mortalidad considerada. Mientras que la neonatal tardía y la postneonatal, fueron las que descendieron más en la primera mitad, en el segundo periodo fueron la neonatal precoz y neonatal tardía. En la neonatal tardía y la postneonatal se ha producido una clara reducción en el porcentaje de descenso: en la primera de un 9.54\% en el periodo de 1975 a 1986 se ha pasado a un $3,33 \%$ en el periodo de 1987 a 1998 y en la segunda de un $7,27 \%$ se redujo a un $1,88 \%$ en los mismos periodos.

La figura 2 muestra cómo la mortalidad infantil se ha reducido en todos sus componentes entre el primer quinquenio y último, pero los porcentajes que suponen los componentes de mortalidad neonatal tardía y postneonatal sobre la mortalidad infantil fueron superiores en 1995-1998 respecto a 19751979: se ha pasado de $14,72 \%$ a $17,93 \%$ y de $27,65 \%$ a 34,59 respectivamente, para la mortalidad neonatal tardía y la postneonatal en los quinquenios comparados.

En la figura 3 se presentan las distribuciones porcentuales de las causas de mortalidad 
Tabla 1

Mortalidad infantil en Andalucía, 1975-1998. Tasas por 1000 nacidos vivos

\begin{tabular}{|c|c|c|c|c|c|c|c|c|c|}
\hline \multirow[b]{2}{*}{ Años } & \multirow[b]{2}{*}{ Nacidos vivos } & \multicolumn{2}{|c|}{ Mortalidad Infantil } & \multicolumn{2}{|c|}{ Neonatal Precoz } & \multicolumn{2}{|c|}{ Neonatal Tardía } & \multicolumn{2}{|c|}{ Postneonatal } \\
\hline & & Número & Tasa & Número & Tasa & Número & Tasa & Número & Tasa \\
\hline 1975 & 124317 & 2693 & 21,66 & 1446 & 11,63 & 468 & 3,76 & 779 & 6,27 \\
\hline 1976 & 126555 & 2426 & 19,17 & 1377 & 10,88 & 333 & 2,63 & 716 & 5,66 \\
\hline 1977 & 124265 & 2088 & 16,80 & 1210 & 9,74 & 318 & 2,56 & 560 & 4,51 \\
\hline 1978 & 122741 & 2009 & 16,37 & 1218 & 9,92 & 263 & 2,14 & 528 & 4,30 \\
\hline 1979 & 118213 & 1879 & 15,90 & 1143 & 9,67 & 251 & 2,12 & 485 & 4,10 \\
\hline 1980 & 116074 & 1522 & 13,11 & 934 & 8,05 & 160 & 1,38 & 428 & 3,69 \\
\hline 1981 & 110337 & 1459 & 13,22 & 912 & 8,27 & 195 & 1,77 & 352 & 3,19 \\
\hline 1982 & 109258 & 1322 & 12,10 & 815 & 7,46 & 179 & 1,64 & 328 & 3,00 \\
\hline 1983 & 104876 & 1216 & 11,59 & 716 & 6,83 & 181 & 1,73 & 319 & 3,04 \\
\hline 1984 & 102050 & 1049 & 10,28 & 636 & 6,23 & 124 & 1,22 & 289 & 2,83 \\
\hline 1985 & 97638 & 994 & 10,18 & 560 & 5,74 & 114 & 1,17 & 320 & 3,28 \\
\hline 1986 & 95039 & 968 & 10,19 & 581 & 6,11 & 116 & 1,22 & 271 & 2,85 \\
\hline 1987 & 93364 & 881 & 9,44 & 477 & 5,11 & 135 & 1,45 & 269 & 2,88 \\
\hline 1988 & 92040 & 847 & 9,20 & 443 & 4,81 & 123 & 1,34 & 281 & 3,05 \\
\hline 1989 & 89562 & 779 & 8,70 & 421 & 4,70 & 122 & 1,36 & 236 & 2,64 \\
\hline 1990 & 89218 & 797 & 8,93 & 397 & 4,45 & 154 & 1,73 & 246 & 2,76 \\
\hline 1991 & 88319 & 738 & 8,36 & 384 & 4,35 & 108 & 1,22 & 246 & 2,79 \\
\hline 1992 & 88012 & 676 & 7,68 & 324 & 3,68 & 139 & 1,58 & 213 & 2,42 \\
\hline 1993 & 86330 & 690 & 7,99 & 332 & 3,85 & 109 & 1,26 & 249 & 2,88 \\
\hline 1994 & 81550 & 535 & 6,56 & 273 & 3,35 & 87 & 1,07 & 175 & 2,15 \\
\hline 1995 & 79422 & 505 & 6,36 & 242 & 3,05 & 92 & 1,16 & 171 & 2,15 \\
\hline 1996 & 77277 & 481 & 6,22 & 232 & 3,00 & 83 & 1,07 & 166 & 2,15 \\
\hline 1997 & 78193 & 420 & 5,37 & 183 & 2,34 & 98 & 1,25 & 139 & 1,78 \\
\hline 1998 & 76627 & 441 & 5,76 & 201 & 2,62 & 67 & 0,87 & 173 & 2,26 \\
\hline \multicolumn{10}{|c|}{ Porcentajes de cambio } \\
\hline $1975-1998$ & & & \begin{tabular}{l|l|}
$-5,62$ \\
\end{tabular} & & \begin{tabular}{|c|}
$-6,38$ \\
\end{tabular} & & $-4,6$ & & $-4,35$ \\
\hline $1975-1986$ & & & $-6,97$ & & $\begin{array}{ll}-6,25 \\
\end{array}$ & & $-9,54$ & & $-7,27$ \\
\hline $1987-1998$ & & & $-5,25$ & & $-6,42$ & & $-3,33$ & & $-1,88$ \\
\hline
\end{tabular}

infantil y sus componentes para los periodos 1975-1979 y 1994-1998. Como puede apreciarse, las causas infecciosas y respiratorias se reducen en el segundo periodo respecto al primero y son sustituidas por las congénitas y perinatales, especialmente en la mortalidad neonatal precoz, pero también en la neonatal tardía y la postneonatal. El porcentaje que suponen las causas perinatales aumentó en la mortalidad neonatal tardía y muy especialmente en la postneonatal. En la primera pasó de un 29,54\% en 1975-1979 a un $44,43 \%$ en 1994-1998, y en la segunda de un
$0,65 \%$ a un $14,32 \%$ entre los mismos periodos. Las causas congénitas aumentaron también aunque en menor proporción y el resto de causas supuso más del 30\% de la mortalidad postneonatal en ambos periodos.

El número de defunciones y las tasas de mortalidad por todas las causas y las específicas por causas perinatales, para la mortalidad infantil y sus componentes, así como las los porcentajes de cambio y las razones de tasas entre 1994-1998 y 1975-1979 se pueden observar en la tabla 2. En el periodo pos- 
Figura 1

Tasas de mortalidad infantil, neonatal precoz, neonatal tardía y postneonatal. Andalucía, 1975-1998

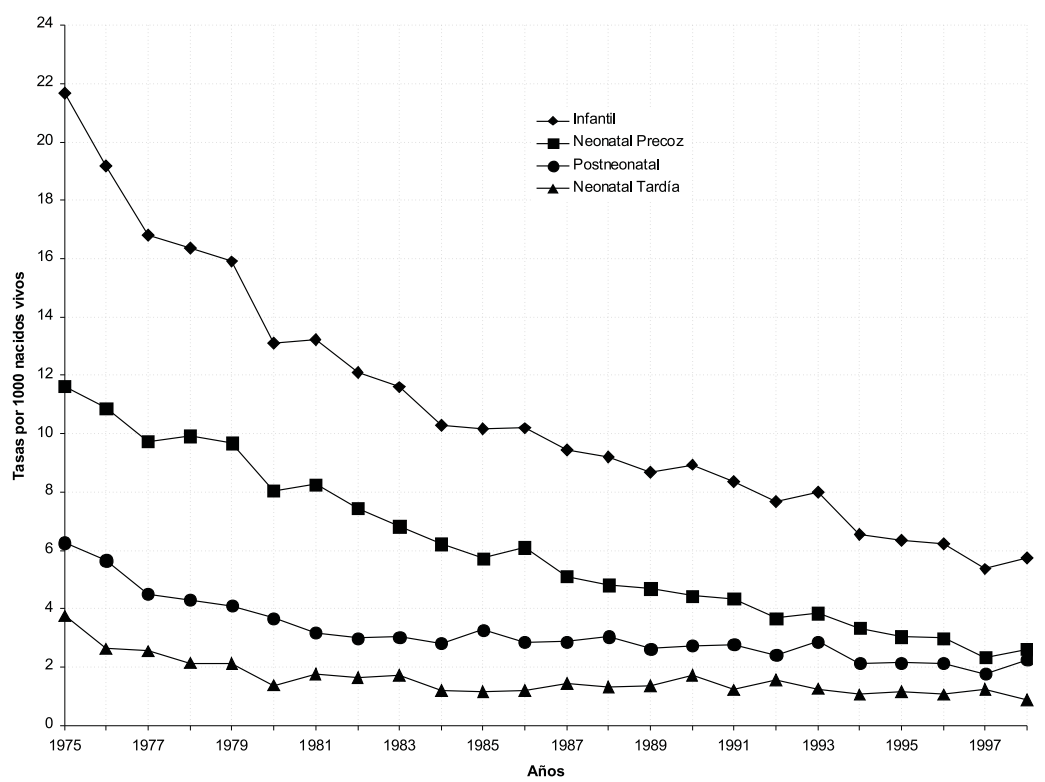

Figura 2

Mortalidad neonatal precoz, neonatal tardía y postneonatal de Andalucía, 1975-1979 y 1994-1998

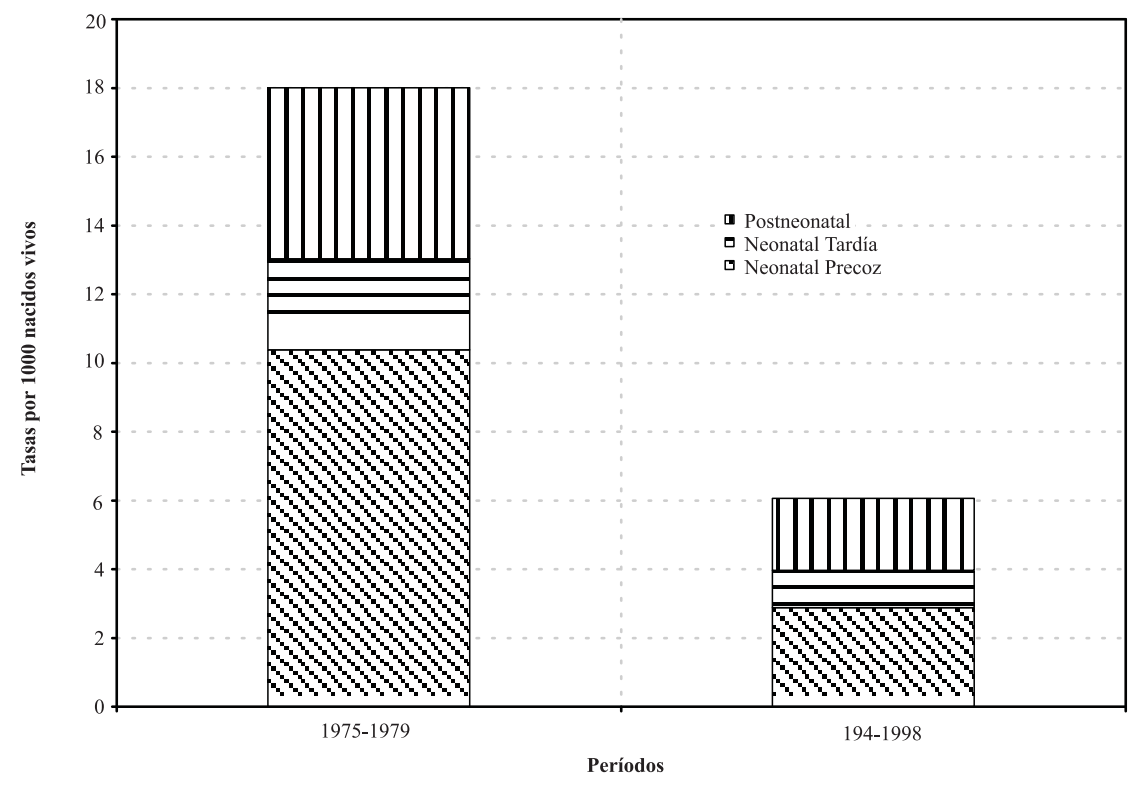


Figura 3

Causas de mortalidad infantil, neonatal precoz, neonatal tardía y postneonatal en Andalucía, 1975-1979 y $1994-1998$

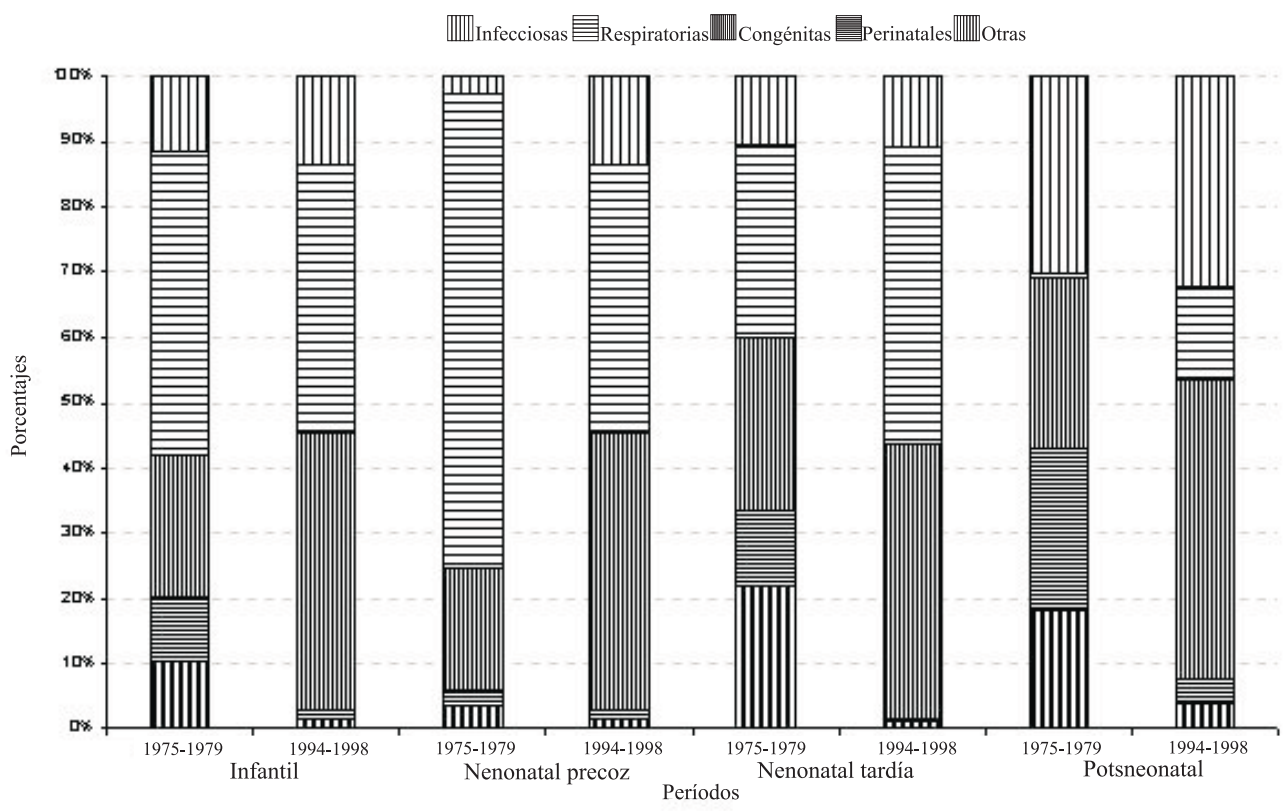

tneonatal la razón de tasas por causas perinatales llega a se10 veces superior en el último quinquenio respecto al primero, este hecho se debe interpretar como un riesgo de morir por causas perinatales diez veces superior en el periodo 1994-1998 que en 1975-1979, asimismo el porcentaje de cambio a lo largo de todo el periodo estudiado aumento en un $61,5 \%$.

En el resto de los componentes de la mortalidad infantil el riesgo de morir por causas perinatales es inferior a uno lo cual quiere decir que el riesgo de morir por estas causas se ha reducido.

\section{DISCUSIÓN}

Tradicionalmente se admite que la mortalidad postneonatal está fuertemente asociada a las condiciones socioeconómicas de la comunidad mientras que la neonatal se ve más influida por la atención al embarazo y parto así como por los cuidados que recibe el neonato. Sin embargo, quizás las nuevas prácticas asistenciales neonatológicas estén modificando no sólo las cifras sino también las causas de mortalidad postneonatal. De hecho, si los cuidados asistenciales alargan el tiempo de vida de un grupo importante de nacidos sin aumentar su supervivencia al año se produce un «traslado» de defunciones del periodo neonatal al postneonatal que se traducirá en un descenso mucho más suave de la mortalidad postneonatal y en una modificación de las causas de defunción de estos nacidos.

Los resultados de este estudio son, en general, consistentes con otras investigaciones ${ }^{6,7}$ que han observado igualmente un enlentecimiento en el descenso de las tasas de mortalidad en el periodo postneonatal llevando a algunos autores a sugerir la existencia de unos límites biológicos por debajo de los cuales sería extremadamente difícil conseguir reducciones, con los conocimientos 
Tabla 2

Tasas de mortalidad por causas perinatales y todas las causa en mortalidad infantil, neonatal precoz, neonatal tardía y postneonatal, razón de tasas entre 1994-1998 y 1975-1979, porcentaje de cambio de las causas perinatales entre 1975-1979 y 1994-1998

\begin{tabular}{|c|c|c|c|c|c|c|}
\hline Períodos & Causas & & Infantil & Neonatal Precoz & Neonatal Tardía & Postneonatal \\
\hline \multirow[t]{4}{*}{$1975-1979$} & Perinatales & Casos & 5153 & 4652 & 481 & 20 \\
\hline & & Tasa & 8,36 & 7,55 & 0,78 & 0,03 \\
\hline & Todas & Casos & 11095 & 6394 & 1633 & 3068 \\
\hline & & Tasa & 18,01 & 7,55 & 2,65 & 4,98 \\
\hline \multirow[t]{4}{*}{ 1980-1984 } & Perinatales & Casos & 3510 & 3070 & 388 & 52 \\
\hline & & Tasa & 6,47 & 5,66 & 0,72 & 0,10 \\
\hline & Todas & Casos & 6568 & 4013 & 839 & 1716 \\
\hline & & Tasa & 12,10 & 7,40 & 1,55 & 3,16 \\
\hline \multirow[t]{4}{*}{ 1985-1989 } & Perinatales & Casos & 1837 & 1539 & 238 & 60 \\
\hline & & Tasa & 3,93 & 3,29 & 0,51 & 0,13 \\
\hline & Todas & Casos & 4469 & 2482 & 610 & 1377 \\
\hline & & Tasa & 9,56 & 5,31 & 1,30 & 2,94 \\
\hline \multirow[t]{4}{*}{ 1990-1993* } & Perinatales & Casos & 1292 & 961 & 242 & 89 \\
\hline & & Tasa & 3,67 & 2,73 & 0,69 & 0,25 \\
\hline & Todas & Casos & 2901 & 1437 & 510 & 954 \\
\hline & & Tasa & 8,24 & 4,08 & 1,45 & 2,71 \\
\hline \multirow[t]{4}{*}{ 1994-1998 } & Perinatales & Casos & 974 & 662 & 194 & 118 \\
\hline & & Tasa & 2,48 & 1,68 & 0,49 & 0,30 \\
\hline & Todas & Casos & 2382 & 1131 & 427 & 824 \\
\hline & & Tasa & 6,06 & 2,88 & 1,09 & 2,10 \\
\hline \multirow[t]{2}{*}{ Razón de tasas } & Perinatales & & 0,30 & 0,33 & 0,63 & 10,00 \\
\hline & Todas & & 0,34 & 0,28 & 0,41 & 0,42 \\
\hline$\%$ de cambio & Perinatales & & $-26,47$ & $-31,05$ & $-9,62$ & $+61,5$ \\
\hline
\end{tabular}

*Este periodo es de cuatro años

científicos actuales. En contra de esta teoría otros científicos argumentan, con lógica, que mientras existan, como fácilmente se observa, diferencias geográficas y socioeconómicas en la distribución de la mortalidad infantil ${ }^{16-20}$ en conjunto y en sus diferentes tramos, se pueden conseguir reducciones que equiparen las tasas de los grupos con valores más y menos elevados.

El aumento en el periodo postneonatal de las tasas de mortalidad por afecciones originadas en el periodo perinatal, se ha puesto de manifiesto en otros trabajos nacionales6 e internacionales ${ }^{20}$. Una vez descartada que sea un artefacto producido por el paso de la octava a la novena revisión de la CIE, como reflejan los resultados de este trabajo con un aumento paulatino a lo largo de los periodos de la serie analizada, cuestiona la interpretación clásica de la mortalidad postneonatal. Analizando las causas que están produciendo las muertes en este periodo, habría que relacionarla más con problemas como el bajo peso al nacer o embarazos de alto riesgo que con causas exógenas responsables de estas muertes en otras épocas ${ }^{21}$.

Otro de los aspectos que implícitamente esta recogido en este trabajo y en los trabajos realizados con los datos procedentes de las estadísticas oficiales, es la subnotificación de la mortalidad infantil. Distintos estudios han evaluado el problema del infrarregistro en España ${ }^{22-24}$, poniendo de manifiesto su importancia, especialmente de las muertes 
de menos de 24 horas, que lógicamente afecta a la mortalidad neonatal precoz. Este problema no tenía mayor trascendencia cuando en las cifras de mortalidad infantil las muertes postneonatales representaban un porcentaje elevado, pero con los valores actuales las muertes de los menores de 24 horas suponen hasta un $50 \%$ del total de las defunciones neonatal precoz y éstas a su vez la mitad de la mortalidad de los menores de un año. Con lo cual es razonable pensar que las cifras de mortalidad neonatal son, en realidad, superiores a las publicadas tanto en Andalucía como en España y aun con la subnotificación existente son superiores a las de otros países de nuestro entorno ${ }^{8}$. Por ello, son posibles reducciones sobre los valores actuales a través de actividades preventivas y asistenciales que mejoren la atención al embarazo, parto y recién nacido ya que las causas que están provocando estas muertes son fundamentalmente congénitas y perinatales.

Para corregir por un lado el subregistro de la mortalidad infantil y disponer de una buena estimación de las cifras reales de mortalidad infantil, y por otro, completar información sobre variables fundamentales como bajo peso al nacer que falta en las estadísticas oficiales de mortalidad sería importante poder tener información de otras fuentes de complementarias que permitiría realizar un seguimiento de la mortalidad y sus causas durante el primer años de vida, imprescindible entre otras para evaluar las actividades de promoción, prevención y asistencia sanitarias que se están realizando.

De todas maneras, los resultados de este trabajo ponen de manifiesto que la mortalidad infantil en conjunto y cada uno de sus componentes entre 1975 y 1998 en Andalucía ha descendido de una manera significativa incluso con porcentajes de descenso superiores al ocurrido en otros países en periodos de tiempo más amplios16, lo que permite cierto optimismo para los próximos años.

\section{BIBLIOGRAFÍA}

1. Anand K, Kant S, Kumar SK. «Development» is not essential to reduce infant mortality rates in India: Experience from Ballabgarh project. J Epidemiol Community Health 2000; 54: 247-53.

2. Mckeown T. El papel de la medicina ¿Sueño, espejismo o némesis? Méjico: Siglo XXI; 1982.

3. García Gil C, Cortés Majó M, Nieto García A, Rosado Martín M, Nájera E. Epidemiological appraisal of the active role of women in the decline of infant mortality in Spain during the twentieth century. Soc Sci Med 1989; 29:1351-1362.

4. Euroestat informe estadístico 1999. Bruselas: Euroestat; 2000.

5. Llanos de la Torre Quiralte M, Garijo Ayestaran M, Poch Olive ML. Evolución de la mortalidad infantil de la Rioja (1980-1998). An Esp Pediatr 2001 Nov; 55(5): 413-20.

6. Gutiérrez JL, Regidor E. Evolución de la mortalidad en el primer año de vida en España (19751988). Gac Sanit 1993; 7:110-5.

7. Rodríguez L, Botting B. Recent trend in postneonatal mortality in England. Population Trend 1989; 55: 7-17

8. Nolte H, Brand A, Koupilová I, McKee M. Neonatal and posneonatal mortality in Germany since unification. J Epidemiol Community Health 2000; 54:84-90.

9. Arroyo Pérez A, Calot G, Fernández Cordón JA. Un siglo de demografía en Andalucía. Sevilla: IEA; 1999.

10. Sociedad Española de Salud Pública y Administración Sanitaria. La Salud Pública ante los desafíos de un nuevo siglo. Granada: Escuela Andaluza de Salud Pública; 2000.

11. INE. Movimiento Natural de la Población Española. Defunciones según causa de muerte. Tomo II. Años 1975-1991. Madrid. Instituto Nacional de Estadística, 1979-1995.

12. IEA. Movimiento Natural de la Población. Defunciones en Andalucía años1992-1998. Sevilla: Instituto de Estadística de Andalucía, 1993-1999.

13. OPS. Clasificación Estadística Internacional de Enfermedades y Problemas Relacionados con la Salud. Tomo III. Washington: OPS; 1999.

Rev Esp Salud Pública 2003, Vol. 77, N. ${ }^{\circ} 3$ 
14. Breslow NE, Day EN. Statistical Methods in Cancer Research, Volumen II. The design of cohort studies. Lyon: IARC; 1987.

15. Klebba AJ. Estimates of selected comparability ratios based on dual coding of 1976 death certificates by eighth and nienth revision of the International Clasification of Diseases. National Vital Statistics Report; vol 28 no 11, 1980.

16. Rosano A, Botto LD, Botting B, Mastroicovo P. Infant mortality and congenital anomalies from 1950 to 1994: an international perspective. J Epidemiol Community Health 2000; 54: 660-6.

17. Terra de Souza AC, Cufino E, Peterson KE, Gardner J, Vasconcelos do Amaral I, Ascherio A. Variations in infant mortality rates among municipalities in the state of Ceará, Northeast Brazil: an ecological analysis. Int J Epidemiol 1998; 28:267-75.

18. Wagstaff A. Socioeconomic inequalities in child mortality: comparisons across nine developing countries. Bull World Health Org 2000; 78: 19-29.

19. Domínguez Berjón MF, Benach J, García Arcal $\mathrm{MD}$, Borrell C. Infant and perinatal mortality in
Spain 1981-1991: Interprovincial variations in $\mathrm{Au}-$ tonomous Communities with extreme economic levels. Eur J Epidemiol 1999; 15: 723-9.

20. Friede A, Rhodes PH, Guyer B, Binkin NJ Hannan MT, Hogue CJ. The postponement of neonatal death into the postneonatal period: evidence from Massachusetts. Am J Epidemiol 1988; 127(1): 161-70.

21. Kirby RS. Neonatal and postneonatal mortality: useful constructs or outdated concepts? J Perinatol 1993 Nov-Dec;13(6):433-41

22. Castilla Catalán J, Moreno Iribas C, Eguino Sasiaín E. Validez de las estadísticas de mortalidad infantil en Navarra. Gac Sanit 1992; 6: 153-6.

23. Mosquera Tenreiro C, González Rico M. Calidad del registro de muertes perinatales. Asturias, 198690. Gac Sanit 1994; 8:112-6.

24. Ferrando J, Borrel C, Ricart M, Plasència A. Infradeclaración de mortalidad perinatal: experiencia de 10 años de vigilancia activa en Barcelona. Med Clin (Barc) 1997; 108: 330-5 